\title{
Mitochondrial function: use it or lose it
}

\author{
J. A. Hawley • S. J. Lessard
}

Published online: 6 February 2007

(C) Springer-Verlag 2007

\section{Introduction}

Type 2 diabetes is a progressive metabolic disorder that develops as a result of both environmental and undefined genetic factors [1]. While the precise molecular aetiology of this disorder remains elusive, there is general consensus that type 2 diabetes has several hallmark features: (1) a breakdown in lipid and carbohydrate metabolism, reflected by elevated levels of circulating metabolites and culminating in excessive deposition of fat in various tissues [2]; (2) skeletal muscle insulin resistance [3]; and (3) coordinated defects in oxidative metabolism [4-6]. In conjunction with these altered metabolic states is the impaired insulin signalling observed in muscle of patients with type 2 diabetes [7]. At present, it is unclear whether these (and other) defects are a consequence of the diabetic state or are instrumental in its development. Unravelling this puzzle is made more difficult because these impairments do not occur in isolation but typically co-exist in the same individual.

\section{Skeletal muscle oxidative phosphorylation and mitochondrial function}

Skeletal muscle is richly endowed with mitochondria and is heavily reliant on oxidative phosphorylation for regenerating ATP from carbohydrate- (predominantly glucose and glycogen) and fat-based fuels (mainly fatty acids and

\section{J. A. Hawley $(\square) \cdot$ S. J. Lessard}

Exercise Metabolism Group, School of Medical Sciences, RMIT University,

P.O. Box 71, Bundoora 3083 VIC, Australia

e-mail: john.hawley@rmit.edu.au ketone bodies). Oxidative phosphorylation is the term used to describe the formation of ATP from ADP and inorganic phosphate $\left(\mathrm{P}_{\mathrm{i}}\right)$ in association with the transfer of electrons from fuel molecules to coenzymes and finally to $\mathrm{O}_{2}$. The dynamic range for the change in the rate of ATP utilisation is $>100$-fold in skeletal muscle [8], while there is a large excess in the ATP production capacity, which allows for 5 20 -fold increases in ATP turnover during exercise [9]. Mitochondrial oxidative phosphorylation provides up to $90 \%$ of cellular ATP, with the balance coming from substrate-level phosphorylation, such as glycolysis.

\section{Muscle oxidative capacity and type 2 diabetes}

A decade ago, Kelley et al. [10] reported an impairment of insulin-stimulated glucose phosphorylation in skeletal muscle of patients with type 2 diabetes. Soon after, Simoneau and Kelley [11] showed that the ratio between the maximal activities of glycolytic (hexokinase) and oxidative (citrate synthase) enzymes in skeletal muscle of individuals with type 2 diabetes was different from that in skeletal muscle of obese or lean glucose-tolerant subjects. At the time, it was suggested that a mismatch between mitochondrial oxidative capacity and the capacity for glycolysis may be an important factor in the development of insulin resistance [11]. In 2002, Kelley et al. [12] reported that the overall capacity of the electron transport chain (estimated using the activity of rotenone-sensitive NADH: $\mathrm{O}_{2}$ oxidoreductase) was reduced by $40 \%$ in skeletal muscle from type 2 diabetic patients compared with that in muscle from healthy volunteers. In a series of studies, Shulman and colleagues have demonstrated reduced mitochondrial density [13], lower rates of mitochondrial phosphorylation [6] and decreased insulin-stimulated ATP synthesis and phosphate 
transport [14] in muscle from insulin-resistant individuals and/or type 2 diabetic subjects. Accordingly, the notion that mitochondrial dysfunction [6, 12-16] and/or impaired oxidative phosphorylation/metabolism [4, 5, 17] contributes to skeletal muscle insulin resistance has gained the ascendancy. However, the precise mechanism by which decreased rates of substrate oxidation in muscle may impair insulin-stimulated glucose transport remains unclear. It has been postulated that mitochondrial dysfunction leads to a reduction in the volume of lipid targeted for oxidation, thereby promoting the accumulation of fatty acids and their metabolites in skeletal muscle [18]. Increased intracellular levels of specific lipid metabolites (such as fatty acyl coenzyme A species, ceramide and diacylglycerol) have been functionally linked to impaired insulin-stimulated glucose uptake in skeletal muscle [19], and provide an indirect mechanism by which impaired mitochondrial lipid oxidation may cause insulin resistance.

The basis of the apparent mitochondrial dysfunction associated with insulin resistance is also yet to be identified. Impairments in muscle oxidative capacity could potentially arise from a defect in mitochondrial function and/or a reduction in the number of mitochondria. In this issue of Diabetologia, Boushel et al. [20] provide direct evidence to refute the former premise by demonstrating that mitochondrial function is normal in the skeletal muscle of patients with type 2 diabetes.

\section{Study findings}

The major finding of the study undertaken by Boushel et al. [20] was that when $\mathrm{O}_{2}$ flux was normalised for markers of mitochondrial content (such as mitochondrial DNA content or oxidative enzyme activity), oxidative phosphorylation and electron transport capacity in muscle extracts obtained from patients with type 2 diabetes were not different from those observed in muscle of age-matched healthy control subjects. In other words, the authors found no evidence for a decrease in the quality of the mitochondria in skeletal muscle from type 2 diabetic subjects. This is an important observation, but one that seems incongruous with reports from several other laboratories describing mitochondrial dysfunction in skeletal muscle from patients with type 2 diabetes [6, 12, 15-17]. However, we suggest that the findings from Boushel et al. [20] and others are not incongruent, but instead should be viewed as inter-related pieces of the same puzzle.

A key feature of the data presented by Boushel et al. [20] was that mitochondrial respiration was indeed lower in the muscle of patients with type 2 diabetes than in the healthy control group, but this was due to a reduction in muscle oxidative capacity (i.e. the number of mitochondria) rather than mitochondrial function itself. This finding highlights the importance of nomenclature when discussing aspects related to muscle mitochondrial function: a true mitochondrial 'dysfunction' implies an inherent abnormality within the mitochondrial machinery rather than a decline in mitochondrial number or density (mass per unit volume). One question that remains to be answered is: 'Do individuals with type 2 diabetes have a genetic predisposition to low muscle oxidative capacity, or does impaired mitochondrial capacity arise from environmental/lifestylerelated factors?' Given the rapidly increasing prevalence of type 2 diabetes over the past decade, we feel the latter is the more likely scenario.

\section{Contractile activity and muscle oxidative capacity: the missing link}

Boushel et al. [20] contend that the missing link connecting muscle mitochondrial function with metabolic disease risk

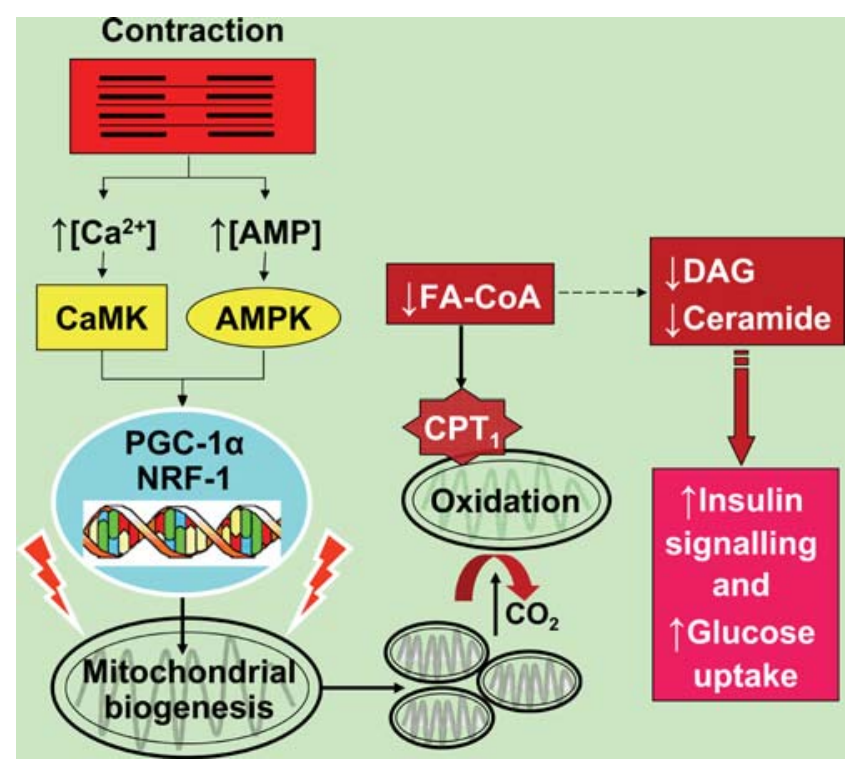

Fig. 1 Potential mechanism by which exercise training-induced enhancement of skeletal muscle oxidative capacity may improve insulin sensitivity. Muscle contraction increases the intracellular concentrations of calcium ions $\left(\left[\mathrm{Ca}^{2+}\right]\right)$ and AMP, which in turn activate the calcium/ calmodulin-dependent protein kinase (CaMK) and AMP-activated protein kinase (AMPK) signalling pathways, respectively. The activation of these signalling cascades increases the expression of nuclear transcription factors such as peroxisome proliferator-activator receptor- $\gamma$ coactivator- $1 \alpha$ (PGC- $1 \alpha)$ and nuclear respiratory factor-1 (NRF-1). These nuclear factors induce the transcription of mitochondrial proteins that promote mitochondrial biogenesis, resulting in increased mitochondrial density in skeletal muscle. Increased mitochondrial density increases the muscle's oxidative capacity, allowing for the shuttling of fatty acyl coenzyme A (FA-CoA) into the mitochondria for oxidation, thus preventing the accumulation of other fatty acid species such as diacylglycerol (DAG) and ceramide, which are known to impair insulin signalling and glucose uptake. $C P T_{1}$, carnitine palmitoyltransferase I 
factors is the level of habitual contractile activity. We agree! Chronic inactivity (or disuse) results in a decrease in mitochondrial number [21], aberrant lipid handling [2] and an impaired health profile [22]. In contrast, chronic activity (exercise training) results in marked mitochondrial biogenesis [21], increased lipid oxidation and turnover [23], and an improved health status [22]. It therefore follows that interventions that result in either a decrease or increase in mitochondrial content should cause reciprocal changes in health/disease outcomes. Several lines of evidence suggest that this is indeed the case. For example, decreased physical activity precipitates a rapid and synchronous decline in insulin-stimulated skeletal muscle glucose uptake, insulin signalling and GLUT4 protein [24], while simultaneously increasing triacylglycerol synthesis in adipose tissue [25]. In sedentary individuals with insulin resistance or type 2 diabetes, the expression of multiple genes involved in aerobic metabolism is downregulated $[4,5]$. In contrast, the age-related declines in the rates of muscle fat oxidative capacity and whole-body insulin sensitivity can be prevented by exercise training [26], while exercise training in patients with type 2 diabetes improves insulin sensitivity by the same magnitude as in healthy control subjects [27]. Of note was that the best predictor of insulin sensitivity in the study of Bruce et al. [27] was muscle oxidative capacity [28]. Exercise training improves the capacity for mitochondrial fatty acid uptake and oxidation while concomitantly reducing muscle lipid content and changes in the saturation status of specific lipids [23]. Indeed, the mitochondrial biogenesis induced by repeated contractile activity provides the most likely mechanism for the enhanced insulin action observed with exercise training in patients with type 2 diabetes [27] and obese individuals [23] (Fig. 1).

Given the strong evidence for a direct role of exercise training in preventing insulin resistance [22], improving glucose tolerance and insulin action in individuals with insulin resistance and type 2 diabetes [29], and promoting mitochondrial biogenesis [21], the question of why such a potent modulator of these conditions is not more commonly prescribed is perplexing and should be of utmost concern to medical healthcare professionals worldwide [22, 30]. The increased prevalence of type 2 diabetes and its strong association with inactivity is producing an 'exercisedeficient phenotype' in which individuals with a particular combination of disease-susceptible genes (i.e. risk factors) interact with undefined environmental conditions (e.g. level of physical activity) and cross a threshold of biological significance that results in overt clinical conditions [31]. In order to arrest the rising incidence of type 2 diabetes, individuals must be willing to make major lifestyle changes, including an increase in the amount of daily physical activity. The message is loud and clear: use it or lose it.

\section{References}

1. Kahn CR (1994) Banting lecture. insulin action, diabetogenes, and the cause of type II diabetes. Diabetes 43:1066-1084

2. McGarry JD (2002) Banting lecture 2001: dysregulation of fatty acid metabolism in the etiology of type 2 diabetes. Diabetes 51:718

3. DeFronzo RA (1992) Pathogenesis of type 2 (non-insulin dependent) diabetes mellitus: a balanced overview. Diabetologia 35:389-397

4. Mootha VK, Lindgren CM, Eriksson KF et al (2003) PGC-1 $\alpha$-responsive genes involved in oxidative phosphorylation are coordinately downregulated in human diabetes. Nat Genet 34:267273

5. Patti ME, Butte AJ, Crunkhorn S et al (2003) Coordinated reduction of genes of oxidative metabolism in humans with insulin resistance and diabetes: potential role of PGC1 and NRF1. Proc Natl Acad Sci USA 100:8466-8471

6. Petersen KF, Dufour S, Befroy D, Garcia R, Shulman GI (2004) Impaired mitochondrial activity in the insulin-resistant offspring of patients with type 2 diabetes. N Engl J Med 350:664-671

7. Bjornholm M, Zierath JR (2005) Insulin signal transduction in human skeletal muscle: identifying the defects in type II diabetes. Biochem Soc Trans 33:354-357

8. Hawley JA, Hargreaves M, Zierath JR (2006) Signalling mechanisms in skeletal muscle: role in substrate selection and muscle adaptation. Essays Biochem 42:1-12

9. Wilson DF (1994) Factors affecting the rate and energetics of mitochondrial oxidative phosphorylation. Med Sci Sports Exerc 26:37-43

10. Kelley DE, Mintun MA, Watkins SC et al (1996) The effect of NIDDM, and obesity on glucose transport and phosphorylation in skeletal muscle. J Clin Invest 97:2705-2713

11. Simoneau JA, Kelley DE (1997) Altered glycolytic and oxidative capacities of skeletal muscle contribute to insulin resistance in NIDDM. J Appl Physiol 83:166-171

12. Kelley DE, He J, Menshikova EV, Ritov VB (2002) Dysfunction of mitochondria in human skeletal muscle in type 2 diabetes. Diabetes 51:2944-2950

13. Morino K, Petersen KF, Dufour S et al (2005) Reduced mitochondrial density and increased IRS-1 serine phosphorylation in muscle of insulin-resistant offspring of type 2 diabetic parents. $\mathrm{J}$ Clin Invest 115:3587-3593

14. Petersen KF, Dufour S, Shulman GI (2005) Decreased insulinstimulated ATP synthesis and phosphate transport in muscle of insulin-resistant offspring of type 2 diabetic parents. PLoS Med 2: e233

15. Petersen KF, Befroy D, Dufour S et al (2003) Mitochondrial dysfunction in the elderly: possible role in insulin resistance. Science 300:1140-1142

16. Schrauwen-Hinderling VB, Kooi ME, Hesselink MK et al (2007) Impaired in vivo mitochondrial function but similar intramyocellular lipid content in patients with type 2 diabetes mellitus and BMI-matched control subjects. Diabetologia 50:113-120

17. Ørtenblad N, Mogensen M, Petersen I et al (2005) Reduced insulin-mediated citrate synthase activity in cultured skeletal muscle cells from patients with type 2 diabetes: evidence for an intrinsic oxidative enzyme defect. Biochim Biophys Acta 1741:206-214

18. Lowell BB, Shulman GI (2005) Mitochondrial dysfunction and type 2 diabetes. Science 307:384-387

19. Schmitz-Peiffer C (2000) Signalling aspects of insulin resistance in skeletal muscle: mechanisms induced by lipid oversupply. Cell Signal 12:583-594 
20. Boushel R, Gnaiger E, Schjerling P, Skovbro M, Kraunsøe R, Della F (2007) Patients with type 2 diabetes have normal mitochondrial function in skeletal muscle. Diabetologia DOI 10.1007/s00125-007-0594-3

21. Hood DA, Irrcher I, Ljubicic V, Joseph AM (2006) Coordination of metabolic plasticity in skeletal muscle. J Exp Biol 209:2265-2275

22. Hawley JA (2004) Exercise as a therapeutic intervention for the prevention and treatment of insulin resistance. Diabetes Metab Res Rev 20:383-393

23. Bruce CR, Thrush AB, Mertz VA et al (2006) Endurance training in obese humans improves glucose tolerance and mitochondrial fatty acid oxidation and alters muscle lipid content. Am J Physiol Endocrinol Metab 291:E99-E107

24. Kump DS, Booth FW (2005) Alterations in insulin receptor signaling in the rat epitrochlearis muscle upon cessation of voluntary exercise. J Physiol 562:829-838

25. Kump DS, Booth FW (2005) Sustained rise in triacylglycerol synthesis and increased epididymal fat mass when rats cease voluntary wheel running. J Physiol 565:911-925

26. Rimbert V, Boirie Y, Bedu M, Hocquette JF, Ritz P, Morio B (2004) Muscle fat oxidative capacity is not impaired by age but by physical inactivity: association with insulin sensitivity. FASEB J 18:737-739

27. Bruce CR, Kriketos AD, Cooney GJ, Hawley JA (2004) Disassociation of muscle triglyceride content and insulin sensitivity after exercise training in patients with type 2 diabetes. Diabetologia 47:23-30

28. Bruce CR, Anderson MJ, Carey AL et al (2003) Muscle oxidative capacity is a better predictor of insulin sensitivity than lipid status. J Clin Endocrinol Metab 88:5444-5451

29. Zierath JR (2002) Invited review: exercise training-induced changes in insulin signaling in skeletal muscle. J Appl Physiol 93:773-781

30. Booth FW, Chakravarthy MV, Gordon SE, Spangenburg EE (2002) Waging war on physical inactivity: using modern molecular ammunition against an ancient enemy. J Appl Physiol 93:3-30

31. Beaudet AL, Scriver CR, Sly WS, Valle D (1995) Genetics, biochemistry, and molecular basis of variant human phenotypes. In: Scriver CR, Beaudet AL, Sly WS et al (eds) The metabolic and molecular bases of inherited disease, vol 1, 7th edn. McGrawHill, New York, pp 79 